\title{
Clinicopathological and Molecular Characteristics of Mammary Adenoid Cystic Carcinoma With Adipocytic Differentiation With Emphasis on the Identification of a Novel $B R A F$ Mutation
}

\author{
GO EUN BAE ${ }^{1}$, NARA YOON ${ }^{2}$, EUN YOON CHO ${ }^{3}, \mathrm{HYUN}^{-S O O ~ \mathrm{KIM}^{4} \text { and SOO YOUN CHO }}$ \\ ${ }^{1}$ Department of Pathology, Chungnam National University School of Medicine, Daejeon, Republic of Korea; \\ ${ }^{2}$ Department of Pathology, The Catholic University of Korea Incheon St. Mary's Hospital, Incheon, Republic of Korea; \\ ${ }^{3}$ Department of Pathology and Translational Genomics, Samsung Medical Center, \\ Sungkyunkwan University School of Medicine, Seoul, Republic of Korea; \\ ${ }^{4}$ Department of Pathology, Severance Hospital, Yonsei University College of Medicine, Seoul, Republic of Korea
}

\begin{abstract}
Background/Aim: Adenoid cystic carcinoma accounts for fewer than $1 \%$ of all breast malignancies. Herein, we describe the clinicopathological characteristics and immunophenotype of mammary adenoid cystic carcinoma that exhibited adipocytic differentiation. Furthermore, we aimed to identify mutations potentially associated with this rare tumor. Case Report: A 52-year-old woman was referred to our Institution after detection of an incidental breast mass. The resected breast specimen contained a well-circumscribed, firm mass surrounded by ill-defined, soft, yellow bulging nodules and measured $2.5 \mathrm{~cm}$ in the greatest dimension. The surgically-resected breast lesion was subjected to pathological examination and immunohistochemical staining. Next-generation sequencing was also carried out on an Ion Torrent Personal Genome Machine, using Ion AmpliSeq Cancer Hotspot Panel v2. Histologically, the tumor had two components: a central area of complex glandular proliferation with solid and cribriform architectures and a peripheral area showing adipocytic differentiation with spindle cell proliferation. Immunohistochemical staining revealed positive immunoreactivity for cytokeratin 5/6, epidermal growth factor receptor, and smooth muscle actin in both the central and peripheral areas. Next-generation
\end{abstract}

Correspondence to: Dr. Soo Youn Cho, Department of Pathology and Translational Genomics, Samsung Medical Center, Sungkyunkwan University School of Medicine, 81, Irwon-ro, Gangnam-gu, Seoul 06351, Republic of Korea. Tel: +82 234102817/+82 234100025, email: sooyoun.cho@samsung.com; Dr. Hyun-Soo Kim, Department of Pathology, Severance Hospital, Yonsei University College of Medicine, 50-1, Yonsei-ro, Seodaemun-gu, Seoul 03722, Republic of Korea. Tel: +82 222281794/+82 23620860, e-mail: hyunsookim@yuhs.ac

Key Words: Breast, adenoid cystic carcinoma, adipocytic differentiation, next-generation sequencing, $B R A F$ mutation, case report. sequencing-based genetic analysis identified a homozygous missense mutation (NM_004333.4:c.1742A>G; p.N581S) in the $v$-Raf murine sarcoma viral oncogene homolog $B(B R A F)$ gene. Conclusion: We report the first case, as far as we are aware, of primary mammary adenoid cystic carcinoma with adipocytic differentiation. A novel missense mutation in $B R A F$ was detected. The potential response to BRAF inhibitors in the presence of this mutation remains a matter for further studies.

Adenoid cystic carcinoma is a rare malignant tumor that most commonly affects major and minor salivary glands (1). It is histopathologically characterized by a biphasic pattern, consisting of a proliferating glandular component and a stromal or basement membrane component. While common in salivary glands, adenoid cystic carcinoma sometimes occurs in the nasopharynx, trachea, skin, lungs, and kidneys.

Ductal and lobular carcinomas account for more than $90 \%$ of breast carcinomas. The remaining $10 \%$ represent a heterogeneous group of histologically rare breast carcinomas with different clinical and pathological characteristics. Mammary adenoid cystic carcinoma is one such rare tumor that occurs in $0.1-1 \%$ of all patients diagnosed with breast carcinoma. It mainly affects women of 50-60 years of age and is usually a triple-negative breast carcinoma expressing neither hormone receptors nor human epidermal growth factor receptor 2 (HER2) (2, 3). Despite being triple-negative breast, mammary adenoid cystic carcinoma is usually an indolent tumor with a favorable outcome when confined to the breast. Lymph node metastasis is very rare $(<2 \%)$, and distant metastases are even exceptional (4). The prognosis of patients with mammary adenoid cystic carcinoma has been reported to be better than that of patients with adenoid cystic carcinoma arising in other locations (5). 
Recently, we encountered a very rare case of mammary adenoid cystic carcinoma showing adipocytic differentiation. Herein, we report its clinical and pathological characteristics and immunophenotype, and we discuss molecular characteristics, with emphasis on a novel v-Raf murine sarcoma viral oncogene homolog B (BRAF) mutation. Comprehensive analysis of the case would expand our knowledge about mammary adenoid cystic carcinoma.

\section{Case Report}

Clinical characteristics. A 52-year-old Korean woman was referred to our Institution because of a non-palpable mass in the left lower breast. The mass was detected during screening ultrasonography at a local clinic. Neither the patient nor her family had any history of breast-related health problems. Initial mammographic series revealed a $1.3 \mathrm{~cm}$-sized indistinct, irregular-shaped, isodense mass in the left lower breast. Magnetic resonance imaging revealed a breast mass with an irregular margin measuring $2.5 \mathrm{~cm}$ in the greatest dimension. After confirmation of the pathological diagnosis by core needle biopsy, surgical excision with sentinel node biopsy was carried out. Two Board-certified pathologists specialized in breast pathology made a final diagnosis of primary adenoid cystic carcinoma of the breast with clear surgical resection margins. The sentinel lymph nodes exhibited no evidence of metastasis. The patient received postoperative adjuvant chemotherapy and radiation therapy. During the 4year follow-up period, the patient remained recurrence-free. The surgically-resected breast lesion was subjected to pathological examination and immunohistochemical staining.

Pathological characteristics. Grossly, the resected breast tissue measured $6.8 \times 5.5 \times 3.1 \mathrm{~cm}$. The cut section showed a well-circumscribed, firm mass surrounded by ill-defined, soft, yellow bulging nodules and measuring $2.5 \mathrm{~cm}$ in the greatest dimension. Histologically, the central portion of the tumor showed complex glandular architectures, composed predominantly of solid or cribriform growth patterns and accompanied by desmoplastic stroma, whereas the peripheral portion of the tumor consisted of solid nests of tumor cells embedded in adipose tissue (Figure 1A). In particular, the central portion displayed cylindromatous neoplastic glands, the lumina of which were filled with basophilic basement membrane-like material characteristic of adenoid cystic carcinoma. The peripherally located yellow nodules showed scattered, small tumor cell nests surrounded by a periductal cuff of cellular myxoid stroma and bound by adjacent adipose tissue. Slender spindle-shaped cells forming the periductal cuff went beyond the boundary and merged imperceptibly with surrounding adipocytes (Figure 1B). These two morphological components appeared to be intermingled.
Immunohistochemistry. The formalin-fixed, paraffinembedded tissue sections were immunostained using a Bond-maX automated immunostainer (Leica Biosystems, Melbourne, Australia) and a Bond Polymer Refined Detection Kit (Leica Biosystems). The primary antibodies targeted estrogen receptor (1:200; Novocastra, Newcastle upon Tyne, UK), progesterone receptor (1:100; Novocastra), HER2 (1:200; Ventana Medical Systems, Tucson, AZ, USA), cytokeratin 5/6 (1:100; Dako, Carpinteria, CA, USA), epidermal growth factor receptor (EGFR; 1:100; Novocastra), smooth muscle actin (SMA; 1:1,000; Dako), and p63 (1:200; Biocare Medical, Concord, CA, USA). Briefly, antigen retrieval was conducted at $97^{\circ} \mathrm{C}$ for $20 \mathrm{~min}$ in ER1 or ER2 buffer. After blocking endogenous peroxidase activity with $3 \%$ hydrogen peroxide for $10 \mathrm{~min}$, primary antibodies were reacted for $15 \mathrm{~min}$ at room temperature. To verify antibody specificity, antimouse immunoglobulin was used instead of the primary antibody as a negative control. Estrogen and progesterone receptor expression status was assessed using the Allred scoring method (6). HER2 expression was evaluated using American Society of Clinical Oncology/College of American Pathologists guideline recommendations (7). For cytokeratin 5/6 immunostaining, staining with a moderateto-strong intensity in the cell membrane was interpreted as positive expression. For SMA and p63 immunostaining, staining with a moderate-to-strong intensity in the cytoplasm and nuclei, respectively, was

Immunohistochemical staining results. We observed a basallike, triple-negative phenotype. The tumor cells were negative for estrogen receptor, progesterone receptor, and HER2. The tumor cells were positive for cytokeratin 5/6 (Figure 1C), SMA (Figure 1D), EGFR, and p63. The peritumoral spindle-shaped cells were positive for cytokeratin 5/6, EGFR, and p63, and the slender spindle-shaped cells within adipose tissue were positive for cytokeratin 5/6, EGFR, and SMA.

Next-generation sequencing. Next-generation sequencing was conducted on an Ion Torrent Personal Genome Machine using Ion AmpliSeq Cancer Hotspot Panel v2 (Life Technologies, Grand Island, NY, USA), as previously described $(8,9)$. The panel contains 2,855 cancer-related mutations in 50 commonly mutated oncogenes and tumor suppressor (please see https://www.thermofisher.com/order/catalog/product/4475346 for complete list). To construct the sequencing library, $10 \mathrm{ng}$ DNA from a formalin-fixed, paraffin-embedded tumor section was subjected to single-tube, multiplex polymerase chain reaction amplification using the Ion AmpliSeq Cancer Primer Pool (Life Technologies) and Ion AmpliSeq Kit reagents (Life Technologies). An Ion 316 Ion Personal Genome Machine chip (Life Technologies) was used. The sample was then treated with $\alpha$-fluoro- $\beta$-ureidopropionic acid reagent to 


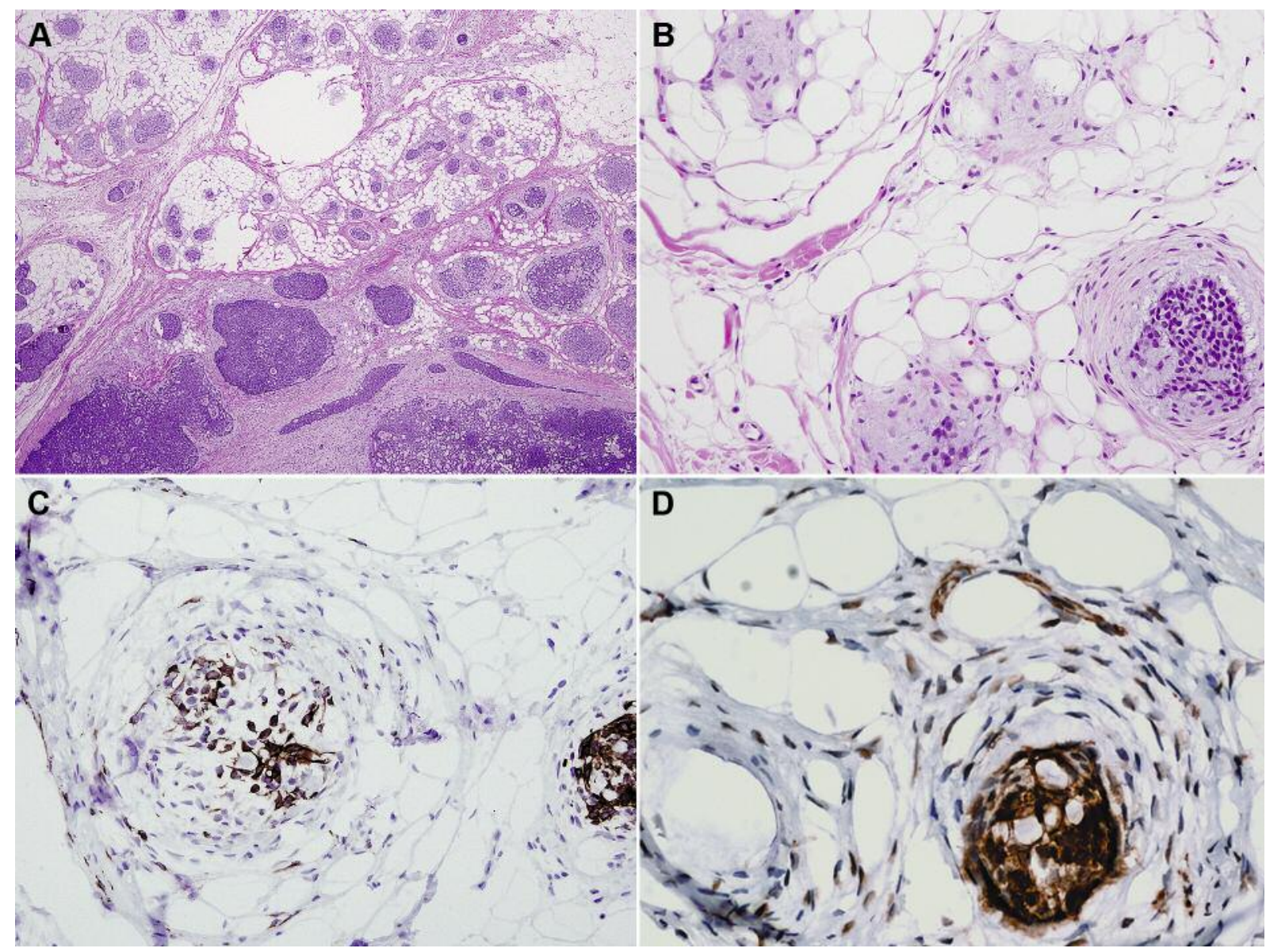

Figure 1. Histopathological features of primary mammary adenoid cystic carcinoma with extensive adipocytic differentiation. A: The tumor tissue exhibited two morphologically distinct components: classic solid and cribriform neoplastic glands in a desmoplastic background (central portion; lower half) and atypical tumor cell nests within adipose tissue (peripheral portion; upper half). B: At the periphery, individual tumor cell nests possessed a periductal cuff of cellular myxoid stroma and adipose tissue (right lower corner). Some nests exhibited spindle-shaped stromal cells only in the remaining myxoid stroma (upper half). A few spindle-shaped cells scattered and merged with adipocytes in the remaining area can be seen. $C$ and D: The tumor nests within the nests, peritumoral spindle-shaped cells, and scattered slender spindle-shaped cells embedded in adipose tissue were positive for cytokeratin $5 / 6(C)$ and smooth muscle actin $(D)$. Staining method: $A$ and $B$, hematoxylin and eosin; $C$ and D, polymer method. Original magnification: A, 40x; B, 100x; C, 100x; D, 200x.

partially digest the primers and phosphorylate the amplicons, which were then ligated to Ion Adapters and purified. Barcoded adapters from the Ion Xpress Barcode Adapters 196 Kit (Life Technologies) were substituted for the nonbarcoded adapter mix supplied in the Ion AmpliSeq Library Kit (Life Technologies). The ligated DNA was subjected to nick translation and amplification to complete the linkage between the adapters and amplicons and to generate sufficient material for downstream template preparation. Two rounds of Agencourt AMPure XP Reagent (Beckman Coulter Diagnostics, Brea, CA, USA) binding at 0.6 and $1.2 \mathrm{bead} / \mathrm{sample}$ volume ratios were used to remove the input DNA and primers from the amplicons. Final library molecules of 125-300 bp were obtained. The library was then transferred to the Ion OneTouch System (Life Technologies) for automated template preparation. Sequencing was carried out on the Ion Personal Genome Machine sequencer (Life Technologies), according to the manufacturer's recommendations. Ion Torrent software version 5.0 was used for automated data analysis, as previously described (9).

Molecular characteristics. Nucleotide sequence alignment revealed a homozygous missense mutation (NM_004333.4: c. $1742 \mathrm{~A}>\mathrm{G}$; p.N581S) in exon 15 of the $B R A F$ gene (allelic frequency, $45.7 \%$; depth of coverage, 644) (Figure 2). 


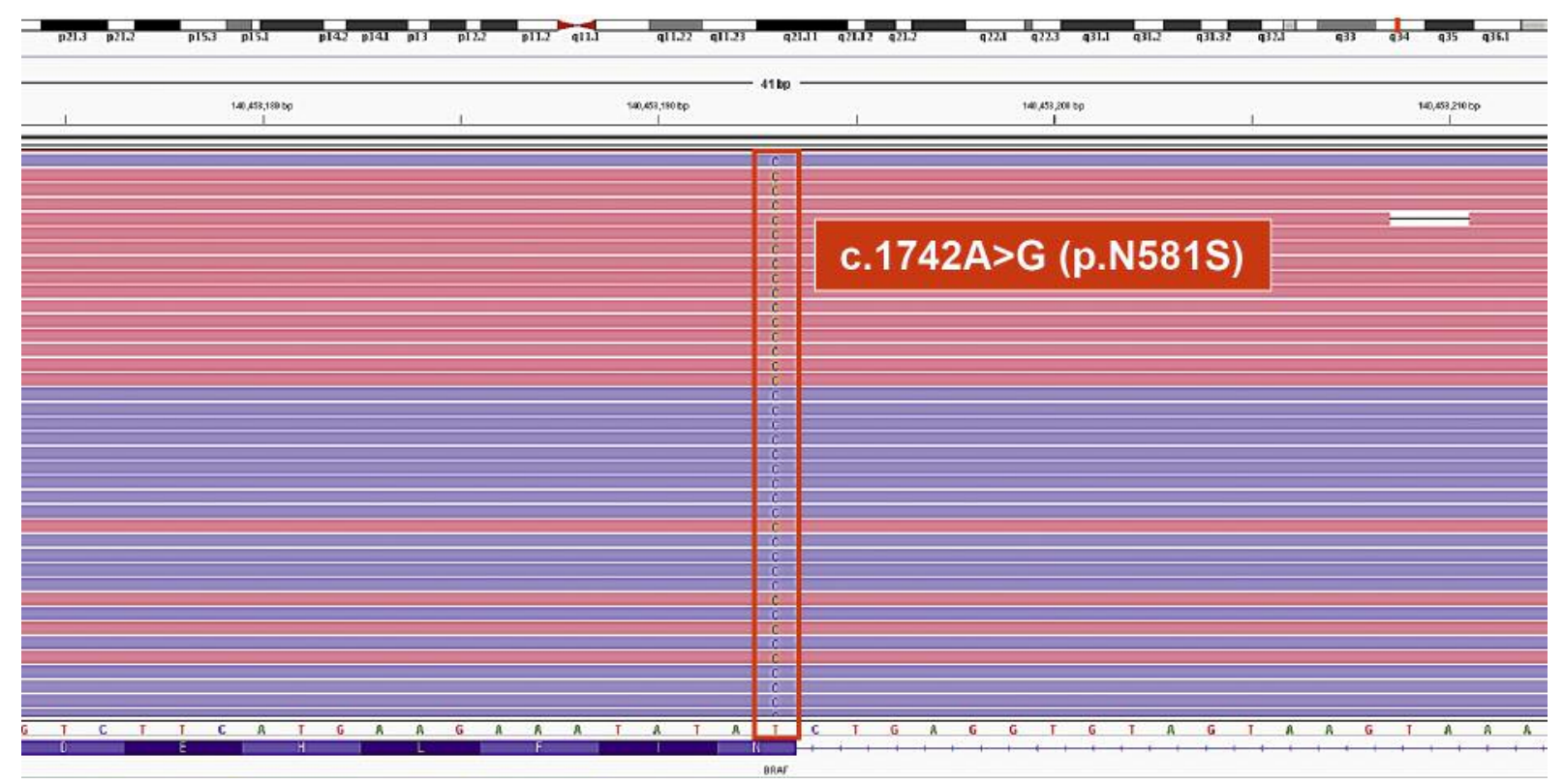

Figure 2. Nucleotide sequence alignment of exon 15 of the v-Raf murine sarcoma viral oncogene homolog $B(B R A F)$ gene, visualized by the Integrative Genomics Viewer. Next-generation sequencing revealed a pathogenic missense mutation in the BRAF gene (NM_004333.4:c.1742A>G; p.N581S) of the patient in this study.

\section{Discussion}

Adenoid cystic carcinoma occurs very rarely in the breast; it accounts for $<1 \%$ of all breast malignancies $(10,11)$. The presence of basement membrane-like material and biphasic cellular pattern of ductal and myoepithelial cells are the most important diagnostic criteria (12-14). Mammary adenoid cystic carcinoma also exhibits a variety of growth patterns, including solid, cribriform, tubular, reticular, and basaloid patterns (15-17). Additionally, foci of squamous differentiation, sebaceous metaplasia, myofibroblastic hyperplasia, adenomyoepitheliomatous differentiation, and syringomatous differentiation have also been documented in mammary adenoid cystic carcinoma (18-20). In this case, we observed peculiar histopathological features of mammary adenoid cystic carcinoma. The individual tumor cell nests had periductal cuffing of cellular stroma and showed a gradual transition to lobular, mature adipose tissue bound by collagenous fibroadipose tissue. The organoid structure of each lobular compartment (tumor cell nests, cellular myxoid stroma, adipose tissue, and fibrous tissue) and the positive immunoreactivities for cytokeratin 5/6, EGFR, and SMA support a neoplastic nature. To the best of our knowledge, this is the first report of a case of primary mammary adenoid cystic carcinoma with adipocytic differentiation.

BRAF, a member of the RAF kinase family, is the most important activator of mitogen-activated protein kinase/ extracellular signal-regulated kinase (ERK) kinase (MEK) in the RAS-RAF-MEK-ERK signaling pathway. Specifically, the mutated BRAF oncoprotein is a major target of inhibitors in various types of malignancies (21). In this study, we identified a pathogenic missense mutation of the BRAF gene, NM_004333.4:c.1742A>G (p.N581S), which to our knowledge has not been previously reported in mammary adenoid cystic carcinoma. To date, pathogenic BRAF mutations have been reported in three cases of mammary adenoid cystic carcinoma. They were all missense mutations [NM_004333.4: c.1391G>A (p.G464E); NM_004333.4:c.1799T>A (p.V600E); NM_ 004333.4:c.1781A $>$ G (p.D594G)] (22). According to the Catalogue Of Somatic Mutations In Cancer database, the incidence of BRAF mutation in mammary adenoid cystic carcinoma is $10.7 \%$ (3/28), which is higher than that reported in conventional breast carcinoma (1.1\%). Even though adenoid cystic carcinomas occurring in diverse organs share similar morphology as well as the genetic alteration of $\mathrm{v}$-myb myeloblastosis viral oncogene homolog-nuclear factor IB (MYB-NFIB) fusion, adenoid cystic carcinomas of the salivary glands $(0.9 \% ; 2 / 209)$ and upper aerodigestive tract $(0 \% ; 0 / 9)$ harbor significantly fewer $B R A F$ mutations than those of the breast, and the frequency of $B R A F$ mutation is much lower than that in breast adenoid cystic carcinoma. The two BRAF mutations observed in salivary adenoid cystic carcinomas were NM_004333.4:c1768G>A (p.V590I) and NM_004333.4: c.1781A>G (p.D594G) (23, 24). Furthermore, adenoid cystic 
carcinoma of the breast usually presents with very few or no recurrent copy number alterations, while adenoid cystic carcinoma of the salivary gland typically harbors multiple recurrent copy number alterations (25-27). Thus, it is reasonable to suggest that the location and environment in which a tumor develops may affect its mutational profile (27).

Relevant genomic aberrations affect cellular functions by altering protein activity and abundance (28). Therefore, the outcome of drug administration (vemurafenib or dabrafenib) inhibiting $B R A F$ kinase differs depending on the location and type of cancer, and on the unique profile of protein expression (29). To define the frequency, durability, and extent of $B R A F$ treatment response in mammary adenoid cystic carcinomas that harbor $B R A F$ mutations, experience and expertise are required.

In summary, we reported the first case of primary mammary adenoid cystic carcinoma with adipocytic differentiation. We described its clinicopathological characteristics and a novel missense mutation of $B R A F$. The potential response to $B R A F$ inhibitors in the presence of this mutation remains to be determined in further studies.

\section{Acknowledgements}

This research was supported by the Basic Science Research Program through the National Research Foundation of Korea funded by the Ministry of Education (2016R1D1A1B03935584) and by a faculty research Grant of the Yonsei University College of Medicine (6-2018-0049).

\section{References}

1 Fordice J, Kershaw C, El-Naggar A and Goepfert H: Adenoid cystic carcinoma of the head and neck: predictors of morbidity and mortality. Arch Otolaryngol Head Neck Surg 125: 149-152, 1999.

2 Coca-Pelaz A, Rodrigo JP, Bradley PJ, Vander Poorten V, Triantafyllou A, Hunt JL, Strojan P, Rinaldo A, Haigentz M Jr. Takes RP, Mondin V, Teymoortash A, Thompson LD and Ferlito A: Adenoid cystic carcinoma of the head and neck - An update Oral Oncol 51: 652-661, 2015.

3 Kim M, Lee DW, Im J, Suh KJ, Keam B, Moon HG, Im SA, Han W, Park IA and Noh DY: Adenoid cystic carcinoma of the breast: A case series of six patients and literature review. Cancer Res Treat 46: 93-97, 2014.

4 Canyilmaz E, Uslu GH, Memis Y, Bahat Z, Yildiz K and Yoney A: Adenoid cystic carcinoma of the breast: A case report and literature review. Oncol Lett 7: 1599-1601, 2014.

5 Miyai K, Schwartz MR, Divatia MK, Anton RC, Park YW, Ayala AG and Ro JY: Adenoid cystic carcinoma of breast: Recent advances. World J Clin Cases 2: 732-741, 2014.

6 Harvey JM, Clark GM, Osborne CK and Allred DC: Estrogen receptor status by immunohistochemistry is superior to the ligand-binding assay for predicting response to adjuvant endocrine therapy in breast cancer. J Clin Oncol 17: 1474-1481, 1999.
7 Wolff AC, Hammond ME, Hicks DG, Dowsett M, McShane LM, Allison KH, Allred DC, Bartlett JM, Bilous M, Fitzgibbons P, Hanna W, Jenkins RB, Mangu PB, Paik S, Perez EA, Press MF, Spears PA, Vance GH, Viale $G$ and Hayes DF: Recommendations for human epidermal growth factor receptor 2 testing in breast cancer: American Society of Clinical Oncology/College of American Pathologists clinical practice guideline update. Arch Pathol Lab Med 138: 241-256, 2014.

8 Ji JH, Oh YL, Hong M, Yun JW, Lee HW, Kim D, Ji Y, Kim DH, Park WY, Shin HT, Kim KM, Ahn MJ, Park K and Sun JM: Identification of driving ALK fusion genes and genomic landscape of medullary thyroid cancer. PLoS Genet 11: e1005467, 2015.

9 Kim S, Lee J, Hong ME, Do IG, Kang SY, Ha SY, Kim ST, Park SH, Kang WK, Choi MG, Lee JH, Sohn TS, Bae JM, Kim S, Kim DH and Kim KM: High-throughput sequencing and copy number variation detection using formalin fixed embedded tissue in metastatic gastric cancer. PLoS One 9: e111693, 2014.

$10 \mathrm{McClenathan} \mathrm{JH}$ and de la Roza G: Adenoid cystic breast cancer. Am J Surg 183: 646-649, 2002.

11 Ghabach B, Anderson WF, Curtis RE, Huycke MM, Lavigne JA and Dores GM: Adenoid cystic carcinoma of the breast in the United States (1977 to 2006): a population-based cohort study. Breast Cancer Res 12: R54, 2010.

12 Azumi $\mathrm{N}$ and Battifora $\mathrm{H}$ : The cellular composition of adenoid cystic carcinoma. An immunohistochemical study. Cancer 60: 1589-1598, 1987.

13 Kasami M, Olson SJ, Simpson JF and Page DL: Maintenance of polarity and a dual cell population in adenoid cystic carcinoma of the breast: An immunohistochemical study. Histopathology 32: 232-238, 1998

14 Anthony PP and James PD: Adenoid cystic carcinoma of the breast: Prevalence, diagnostic criteria, and histogenesis. J Clin Pathol 28: 647-655, 1975.

15 Rosen PP: Adenoid cystic carcinoma of the breast. A morphologically heterogeneous neoplasm. Pathol Annu 24 Pt 2: 237-254, 1989.

16 Ro JY, Silva EG and Gallager HS: Adenoid cystic carcinoma of the breast. Hum Pathol 18: 1276-1281, 1987.

17 Marchio C, Weigelt B and Reis-Filho JS: Adenoid cystic carcinomas of the breast and salivary glands (or 'The strange case of Dr Jekyll and Mr Hyde' of exocrine gland carcinomas). J Clin Pathol 63: 220-228, 2010.

18 Hoda SA, Brogi E, Koerner FC and Rosen PP: Rosen's Breast Pathology. Philadelphia, USA: Lippincott Williams and Willkins, 2014.

19 Van Dorpe J, De Pauw A and Moerman P: Adenoid cystic carcinoma arising in an adenomyoepithelioma of the breast. Virchows Arch 432: 119-122, 1998.

20 Noske A, Schwabe M, Pahl S, Fallenberg E, Richter-Ehrenstein C, Dietel M and Kristiansen G: Report of a metaplastic carcinoma of the breast with multi-directional differentiation: An adenoid cystic carcinoma, a spindle cell carcinoma and melanoma. Virchows Arch 452: 575-579, 2008.

21 Cantwell-Dorris ER, O'Leary JJ and Sheils OM: BRAFV600E: Implications for carcinogenesis and molecular therapy. Mol Cancer Ther 10: 385-394, 2011.

22 Wetterskog D, Wilkerson PM, Rodrigues DN, Lambros MB, Fritchie K, Andersson MK, Natrajan R, Gauthier A, Di Palma S, Shousha S, Gatalica Z, Topfer C, Vukovic V, A'Hern R, Weigelt 
B, Vincent-Salomon A, Stenman G, Rubin BP and Reis-Filho JS Mutation profiling of adenoid cystic carcinomas from multiple anatomical sites identifies mutations in the RAS pathway, but no KIT mutations. Histopathology 62: 543-550, 2013.

23 Fielding GA: Should the lap band be removed to treat pseudoachalasia? Gastroenterol Hepatol (NY) 9: 471-473, 2013.

24 Tetsu O, Phuchareon J, Chou A, Cox DP, Eisele DW and Jordan $\mathrm{RC}$ : Mutations in the $c$-KIT gene disrupt mitogen-activated protein kinase signaling during tumor development in adenoid cystic carcinoma of the salivary glands. Neoplasia 12: 708-717, 2010.

25 Persson M, Andrén Y, Moskaluk CA, Frierson HF Jr., Cooke SL, Futreal PA, Kling T, Nelander S, Nordkvist A and Persson F: Clinically significant copy number alterations and complex rearrangements of $M Y B$ and $N F I B$ in head and neck adenoid cystic carcinoma. Genes, Chromosomes and Cancer 51: 805817, 2012.

26 Horlings HM, Weigelt B, Anderson EM, Lambros MB, Mackay A, Natrajan R, Ng CK, Geyer FC, van de Vijver MJ and ReisFilho JS: Genomic profiling of histological special types of breast cancer. Breast Cancer Res Treat 142: 257-269, 2013.
27 Persson M, Andren Y, Mark J, Horlings HM, Persson F and Stenman G: Recurrent fusion of $M Y B$ and $N F I B$ transcription factor genes in carcinomas of the breast and head and neck. Proc Natl Acad Sci USA 106: 18740-18744, 2009.

28 Lawrence RT, Perez EM, Hernandez D, Miller CP, Haas KM, Irie HY, Lee SI, Blau CA and Villen J: The proteomic landscape of triple-negative breast cancer. Cell Rep 11: 630-644, 2015.

29 Holderfield M, Deuker MM, McCormick F and McMahon M: Targeting RAF kinases for cancer therapy: $B R A F$-mutated melanoma and beyond. Nat Rev Cancer 14: 455-467, 2014.

Received December 3, 2018 Revised December 11, 2018 Accepted December 12, 2018 